\title{
Context-Aware Adaptation of Service Front-Ends
}

\author{
Francisco Javier Caminero Gil ${ }^{1}$, Fabio Paternò ${ }^{2}$, and Jean Vanderdonckt ${ }^{3}$ \\ ${ }^{1}$ TID, Telefónica I+D \\ Distrito Telefónica, Edificio Oeste 1, Planta 5, Ronda de la Comunicación, s/n \\ E-28050 Madrid, Spain \\ fjcg@tid.es \\ ${ }^{2}$ CNR-ISTI, HIIS Laboratory \\ Via Moruzzi 156124 Pisa, Italy \\ fabio.paterno@isti.cnr.it \\ ${ }^{3}$ Université catholique de Louvain \\ Place des Doyens, 1, B-1348 Louvain-la-Neuve (Belgium) \\ jean.vanderdonckt@uclouvain.be
}

\begin{abstract}
Ambient Intelligence implies the need for context-aware adaptation of user interfaces. This adaptation with respect to the context of use is applicable to a wide spectrum of interactive applications ranging from front ends of web services, information systems to multimedia and multimodal applications. Although the ultimate goal of this adaptation is always for the ultimate benefit of the end user, many approaches and techniques have been used to various degrees of experience and maturity that effectively and efficiently support context-aware adaptation. This workshop is intended to review the state of the art in this domain, while looking at a broad range of applications, to discuss positive and negative experiences of context-aware adaptation, and to come up with criteria and requirements for driving such adaptation.
\end{abstract}

\section{Theme, Goals, Relevance and Format}

Ambient Intelligence needs the support of multi-device user interfaces, which are sensitive and responsive to people and their behaviors, and able to deliver advanced functions, services and experiences. The context of use can vary in terms of many aspects: users, technology, environment, social relations. Many different pieces of work have been conducted in order to address the challenge of context-aware adaptation with valuable inputs from various disciplines such as, but not limited to ubiquitous computing, pervasive applications, ambient intelligence, artificial intelligence, engineering interactive computing systems, mobile human-computer interaction. These contributions are often expressed in a format that prevents them to compare them to each other, mainly because they are heterogeneous and their format is inconsistent. This workshop is aimed at addressing context-aware adaptation of user interfaces via an interaction model according to three dimensions (adapted from [1]): a descriptive power that consists in characterizing a sufficiently large spectrum of adaptation techniques according to a unified format, an evaluative power that consists in comparing different adaptation techniques based on the same format, and a generative virtue that is intended to identify holes in the resulting design space in order to foster further 
research and development of new interaction techniques. "A good interaction model must strike a balance between generality (for descriptive power), concreteness (for evaluative power) and openness (for generative power)" [1]. This means that there is a need for creating a design space for context-aware adaptation of any kind of user interface that should exhibit enough expressiveness (for guarantying enough descriptive power), decidability (for ensuring the ability to assess any adaptation technique), and flexibility (for accommodating new adaption techniques that were previously unforeseen). There is also a need for discussing and reviewing the state of the art in the domain of UI adaptation under the view-point of context-awareness since most surveys are either obsolete [2] or do not address context-awareness [3]. This state of the art will be namely focusing on models, methods, and tools that support context-aware adaptation of UIs, in particular in order to address existing challenges.

The workshop will be a half day workshop that will result into discussing and reviewing the state of the art in the area of context-aware adaptation: a design space for context-aware adaptation of user interfaces, criteria and requirement for an Adaptation Specification Language (ASL), and a common format for expressing such adaptation rules.

We intend to discuss at the workshop the following topics:

- How can we obtain an ambient intelligence where the user interfaces of multiple devices are sensitive and responsive to people and their behaviors, and able to deliver advanced functions, services and experiences?

- What are the major challenges (e.g., conceptual, methodological, technical, organizational) for developing context-aware adaptation of user interfaces?

- For which kinds of systems or applications are context-aware user interfaces particularly useful?

- When and how could we measure the effectiveness, the efficiency of contextaware adaptation?

- How could we measure the quality of the user interface resulting from a contextaware adaptation process?

- In which ways will context-aware adaptation affect user interfaces in the future and how will they evolve?

- What kinds of context-aware adaptation do you see as particularly promising?

\section{References}

1. Beaudouin-Lafon, M.: Designing interaction, not interfaces. In: Proc. of ACM Working Conf. on Advanced Visual Interfaces AVI 2004, Gallipoli, May 25-28, pp. 15-22. ACM Press, New York (2004)

2. Dieterich, H., Malinowski, U., Kuhme, T., Schneider-Hufschmidt, M.: State of the art in adaptive user interfaces. In: Schneider-Hufschmidt, M., Kuhme, T., Malinowski, U. (eds.) Adaptive User Interfaces Principles and Practice, pp. 13-48. Elsevier Science Publishers B.V., Amsterdam (1993)

3. López-Jaquero, V., Vanderdonckt, J., Montero, F., González, P.: Towards an Extended Model of User Interface Adaptation: The ISATINE Framework. In: Gulliksen, J., Harning, M.B., van der Veer, G.C., Wesson, J. (eds.) EIS 2007. LNCS, vol. 4940, pp. 374-392. Springer, Heidelberg (2008) 